\title{
Cystic dilation of the distal end of the nasolacrimal duct: underrated cause of epiphora in adults and its endoscopic
} treatment* P. Eloy' , A.L. Poirrier², T. Nicoli², C. Marlair', G. Delahaut', E. Leruth',
P. Rombaux'

HNS \& ENT Department, CHU-Mont-Godinne, UCL, Yvoir, Belgium

Royal National Throat Nose and Ear Hospital, London, United Kingdom
Rhinology 50: 436-441, 2012

DOI:10.4193/Rhino12.067

*Received for publication:

April 25, 2012

Accepted: May 30, 2012

\begin{abstract}
Summary
Epiphora is a frequent reason for ophthalmologic consultation. Among the multiple causes, obstructions of the lacrimal excretory system are common. Sacal and postsacal obstructions are much more frequent than presacal obstructions. Obstruction at the level of the Hasner's valve is rare and likely underestimated.

The authors report the clinical history and the imaging of 3 patients with a cystic dilation of the distal end of the nasolacrimal duct (NLD). These patients were easily managed by an ENT surgeon. In one case, the surgery consisted of an endonasal DCR where in the 2 other cases, a marsupialisation of the cystic expansion of the nasolacrimal duct was successfully performed with the microdebrider. The authors review the world literature on this specific topic. They conclude that a coronal sinus CT scan and an inferior meatus endoscopy should be included in the ophthalmologic work-up performed in all cases of low obstruction of the lacrimal system. When there is a dilation of the distal end of the NLD the marsupialisation of the cystic expansion in the inferior meatus is the option of treatment instead of performing a DCR. ENTs must play a role in the assessment and treatment of low obstructions of the lacrimal excretory system.
\end{abstract}

Key words: nasolacrimal duct obstruction, Hasner's valve, dilation of the distal end of the nasolacrimal duct, epiphora, inferior meatus, endonasal surgery, marsupialisation, dacryocystorhinostomy

\section{Introduction}

Adult-onset epiphoras are common complaints in routine ophthalmologic consultations. Among the various causes of epiphora, obstructions of the lacrimal excretory system are relatively common. The obstruction can be presacal, sacal or postsacal where the presacal obstructions are far less frequent than sacal and postsacal obstructions ${ }^{(1)}$.

Obstruction at the level of the Hasner's valve is an uncommon and likely underestimated cause of epiphora in adults ${ }^{(2,3)}$. We present a series of 3 cases where epiphora related to Hasner's valve involvement was successfully treated (endonasally and endoscopically) by an ENT surgeon.

\section{Clinical cases}

CR1

A 65-year-old woman complained of persistent right-sided epiphora. She underwent a successful left-sided endonasal endoscopic dacryocystorhinostomy (DCR) 2 years prior to presentation. On clinical examination there was tear stasis in the right conjunctival lake. Pressure on the medial canthal region did not, however, express pus. Syringing of the right lacrimal system through the inferior canaliculus revealed a reflux of saline in the superior canaliculus. Furthermore, probing of the lacrimal system revealed a 'hard stop' with the medial wall of the lacrimal fossa. She underwent a coronal CT scan, which identified a bul- 

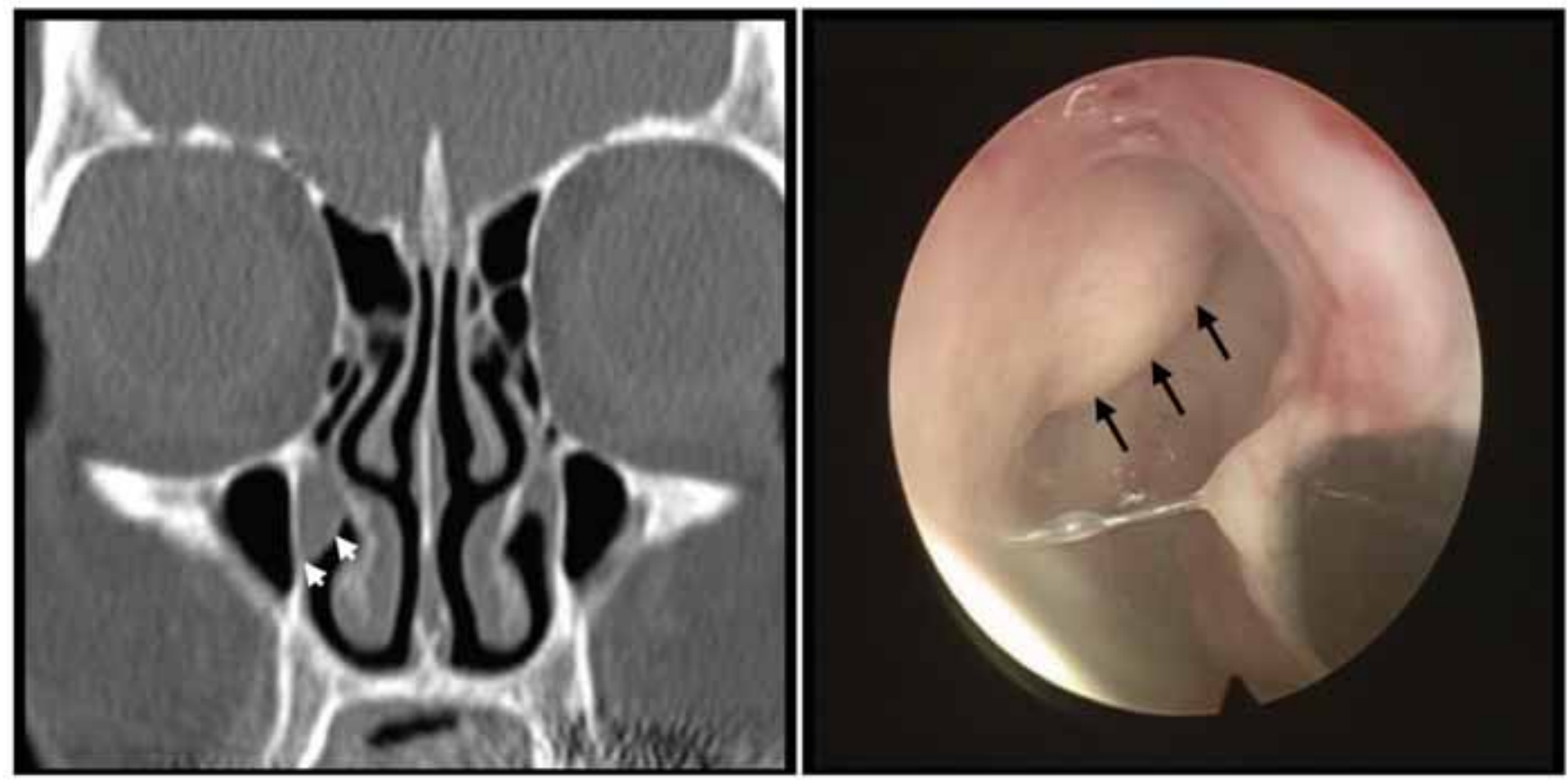

Figure 1. (a) Presence of a buldging in the right inferior meatus coming from the lower end of the nasolacrimal duct - (white arrows). (b) endoscopic view-right inferior meatus- visualization of a cystic lesion in the superior aspect of the meatus - (black arrows).

ging in the inferior meatus (Figure 1a). A consequent dacryocystography confirmed obstruction at the level of the nasolacrimal duct but contrast medium was not seen to fill the expansion at the distal end of the nasolacrimal duct (NLD). Nasal endoscopy demonstrated the presence of a 'cystic lesion' originating from the lower portion of the nasolacrimal duct (Figure 1b) and the diagnosis of a cystic dilation of the NLD was made. An endonasal dacryocystorhinostomy was performed under general anaesthesia in an ambulatory setting. The patient has been free of symptoms for the past 9 months.

CR2

A 35-year-old lady from Georgia presented to our outpatient clinic with left-sided ephiphora on a background of recurrent, persisting dacryocystitis refractory to numerous topical, medical therapies. The clinical examination did not show extrusion of pus from the medial canthal region with finger pressure but probing of the left lacrimal pathway revealed a 'hard stop,' and syringing a reflux of mucoid secretion. A coronal CT scan demonstrated the existence of a large 'cyst' beneath the left inferior turbinate together with dilation of the nasolacrimal duct (Figures $2 \mathrm{a}$ and $2 \mathrm{~b}$ ) and the subsequent dacryocystography confirmed the diagnosis of low obstruction of the lacrimal system. Surgery was conducted under general anaesthesia and endoscopic guidance using a microdebrider. The procedure consisted of a marsupialisation of the lesion in the inferior meatus.
At the end of the procedure, syringing confirmed absence of a stenosis in the high lacrimal excretory system. A silicone intubation catheter (Monocath) was left in situ for 2 weeks and the patient was prescribed steroid eye drops for the same period of time. The patient was free of disease at her 6-month follow-up appointment.

\section{CR3}

A 43-year-old woman presented with right-sided epiphora with no obvious predisposing factors. Syringing confirmed a reflux through the superior lacrimal punctum upon injecting saline via the inferior lacrimal punctum. A coronal CT revealed an expansion of the distal end of the nasolacrimal duct in the inferior meatus (Figures $3 a$ and $3 b$ ). A succeeding nasal endoscopy confirmed the finding of a cystic expansion at the low extremity of the nasolacrimal duct (Figure 3c). Marsupialisation of the lesion was successfully carried out with a microdebrider through the inferior meatus followed by a Monocath (silicone catheter) intubation that was left in place for 2 weeks. The patient has been disease-free for the last 6 months.

\section{Discussion}

Dacryocystocele is an uncommon form of congenital nasolacrimal duct (NLD) obstruction in newborns. Typically the disease is characterized by a triad of a cystic expansion of the NLD beneath the inferior turbinate, dilation of the NLD and lacrimal 

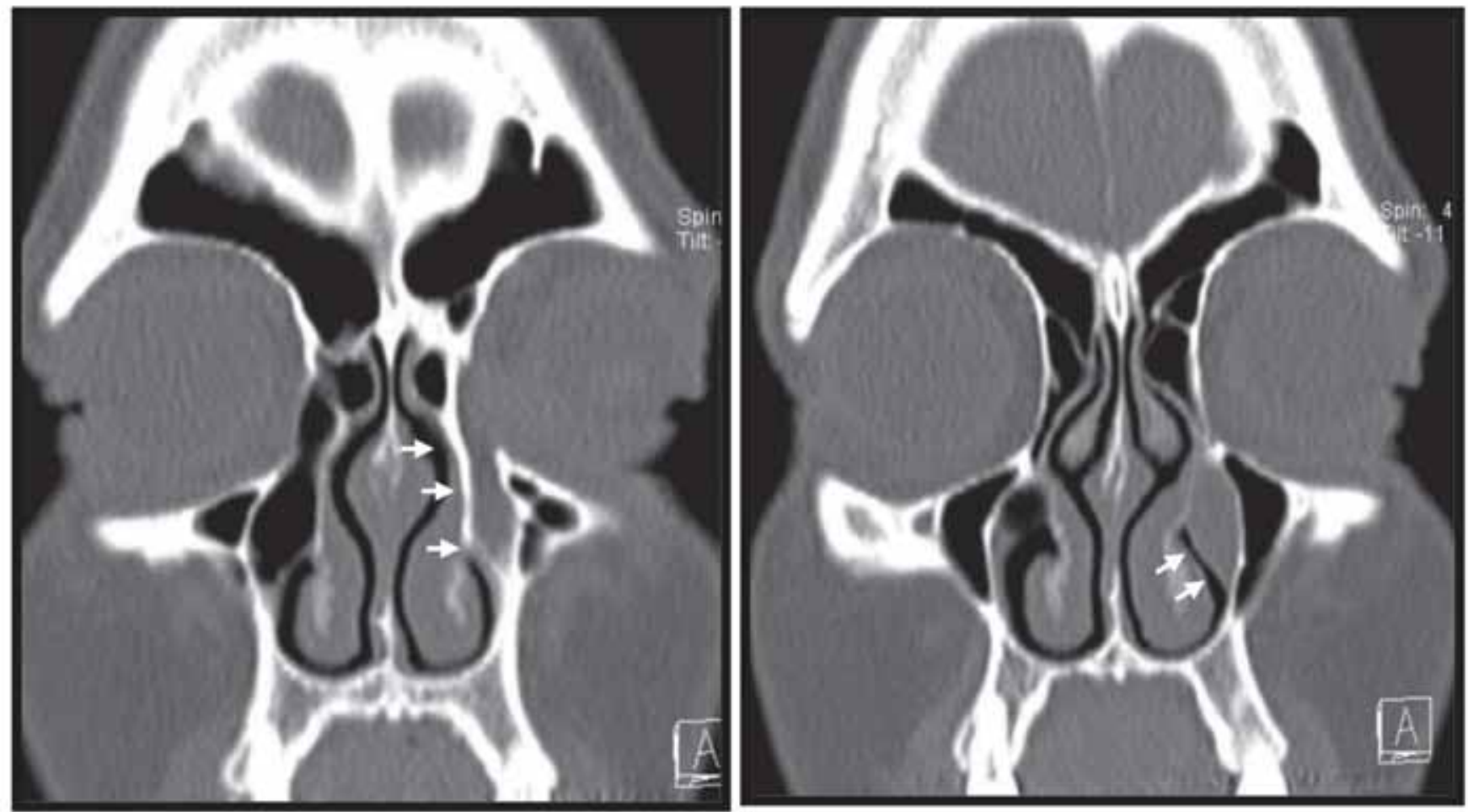

Figure 2. (a) Coronal sinus CT scan - dilation of the left nasolacrimal duct - (white arrows). (b) coronal CT scan - presence of a cystic expansion of the lower end of the nasolacrimal duct beneath the left inferior turbinate - (white arrows).

sac, and a swelling in the medial canthal region. Dacrocystoceles affect neonates in the first weeks of life, are more common in females ${ }^{(4)}$ and can be associated with nasal obstruction when the lesion is large. Respiratory distress in feeding or sleeping has been reported when the disease is bilateral as neonates are obligatory nasal breathers ${ }^{(5-7)}$. When a dacryocystocele is symptomatic, surgery is performed as early in life as possible to reduce the incidence of complications such as dacryocystitis and cellulitis ${ }^{(8)}$. The surgery consists of either probing of the lacrimal system or, preferably, endonasal endoscopic marsupialisation of the cystic lesion with small through-cutting instruments or a microdebrider ${ }^{(9-11)}$.

In contrast, adult-onset low obstructions of the lacrimal drainage pathway are always acquired ${ }^{(12)}$. Most cases are primary and result from inflammation of an unknown cause that leads to occlusive fibrosis.

These obstructions frequently affect middle-aged or elderly females. There is often some connection with menopause but the exact pathogenesis remains unknown. The obstruction is commonly at the junction of the lacrimal sac and the NLD (the valve of Krause) or at the level of the NLD in its inferior portion (the valve of Taillefer) ${ }^{(13,14)}$. A dacryocystorhinostomy (DCR) is the surgical treatment of choice as it bypasses the entire nasolacri- mal duct ${ }^{(1,15,16)}$. Obstruction at the level of the Hasner's valve is uncommon but its incidence is likely to have been underestimated. To date, only one author has published on this topic in the world literature. The first paper included 3 cases similar to ours ${ }^{(2)}$ while the second one incorporated additional cases of stenosis of the Hasner's valve without cystic formation ${ }^{(3)}$.

To make a diagnosis of a low obstruction of the lacrimal pathway, complete ophthalmologic and otolaryngologic assessment and work-up need to be carried out. The ophthalmologic assessment consists of a clinical examination, Jones dye testing, syringing and probing and in some cases imaging of the problem area. The probing typically reveals a 'hard stop' within the medial wall of the lacrimal fossa while the syringing confirms a reflux of saline injected through the inferior lacrimal punctum through the superior one. Digital substraction dacryocystography is the gold standard for visualizing the level of the obstruction ${ }^{(13)}$. In our 3 cases the dacryocystography showed that the obstruction was at the level of the NLD but the contrast medium did not fill the lower end of the NLD.

The ENT evaluation includes a nasal endoscopy and a coronal sinus CT scan.

The nasal endoscopy is nowadays an important part of the otolaryngologic work-up. Before any lacrimal surgery, particularly 

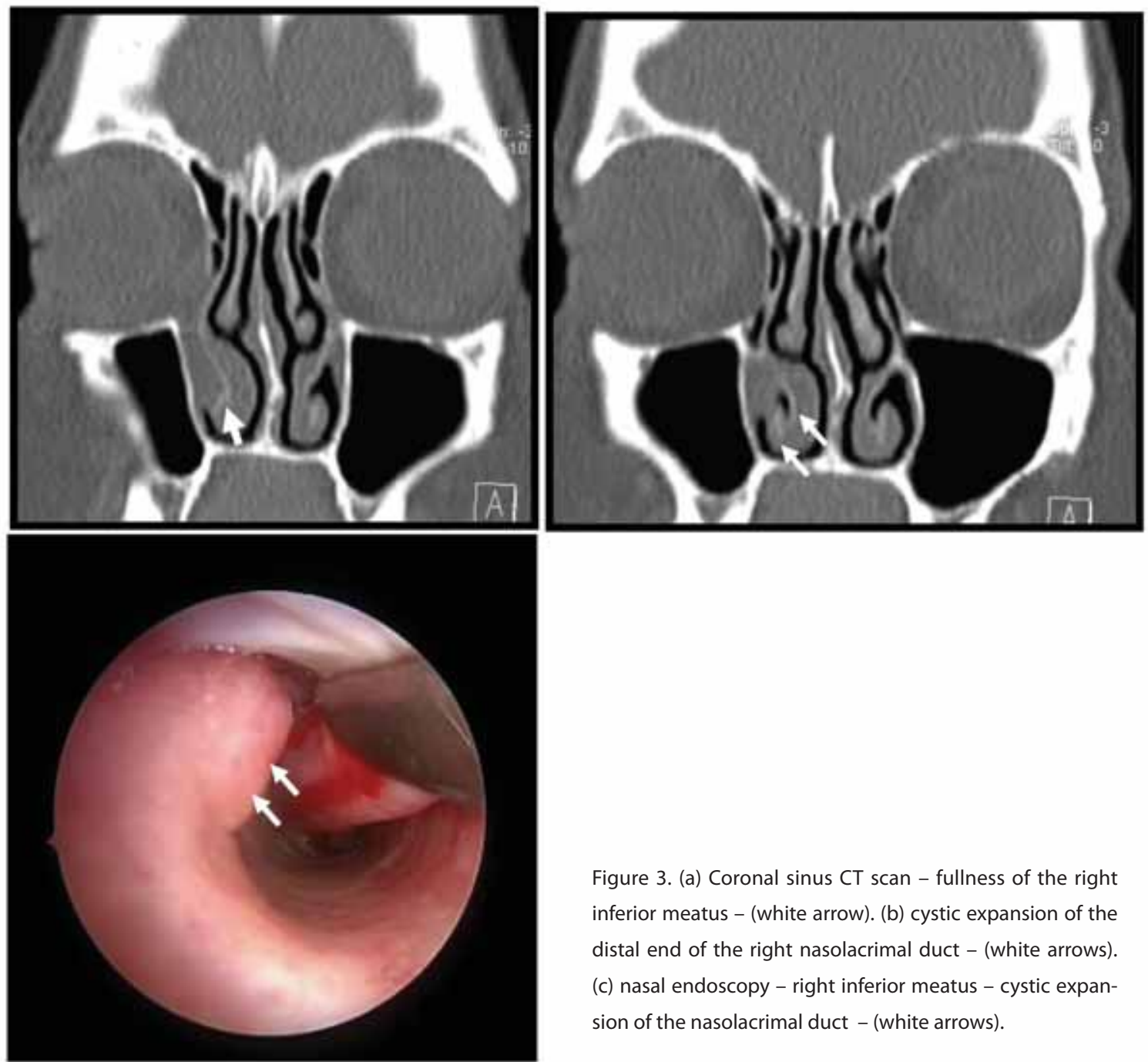

Figure 3. (a) Coronal sinus CT scan - fullness of the right inferior meatus - (white arrow). (b) cystic expansion of the distal end of the right nasolacrimal duct - (white arrows). (c) nasal endoscopy - right inferior meatus - cystic expansion of the nasolacrimal duct - (white arrows).

dacryocystorhinostomy, is carried out, the ENT surgeon must ensure adequate access to the lacrimal eminence and the middle meatus. The surgeon must rule out significant nasal septal deviation, concha bullosa or paradoxical middle turbinate. These abnormalities may justify a different procedure during the same anaesthesia to prevent the development of postoperative adhesions ${ }^{(16)}$.

To make the diagnosis of a stenosis of the Hasner's valve or to visualize a cystic dilation of the distal end of the NLD, an endoscopy of the inferior meatus must be performed ${ }^{(2,3,17)}$. This region is not routinely examined by ENT surgeons. It requires a small rigid or a flexible endoscope and topical anaesthesia because in-fracturing of the inferior turbinate is liable to causing pain to the patient. However, a small lesion located high in the inferior meatus can remain unrecognized with a clinic-based nasal endoscopy. Moreover, it is known from cadaveric dissections that there are different forms of Hasner's valves in normal patients ${ }^{(18)}$. Endoscopic findings must therefore be examined in light of patient's complaints and in unison with data from the ophthalmologic work-up before any surgery is planned.

A sinus CT scan is currently ordered before any endonasal endoscopic surgery from a medicolegal point of view. It provides information about the anatomy of the lateral nasal wall and the presence of a sinus disease. In case of a lacrimal surgery, it gives information about the pneumatisation of the Agger nasi cell and the insertion of the uncinate onto the ascending process of the maxilla. These two structures are important landmarks when performing an endonasal DCR ${ }^{(19)}$. However, $\mathrm{CT}$ sinus scans do not give information about the patency of the lacrimal pathway itself as the NLD can be opaque while being patent. Dacryocystography is therefore, after the Jones dye testing, the best paraclinical investigation to demonstrate the patency or obstruction of the lacrimal system ${ }^{(13)}$.

A CT scan also gives information about the anatomy of the inferior meatus. In our case series, the CT scans demonstrated the cystic dilation of the distal end of the NLD that could not 


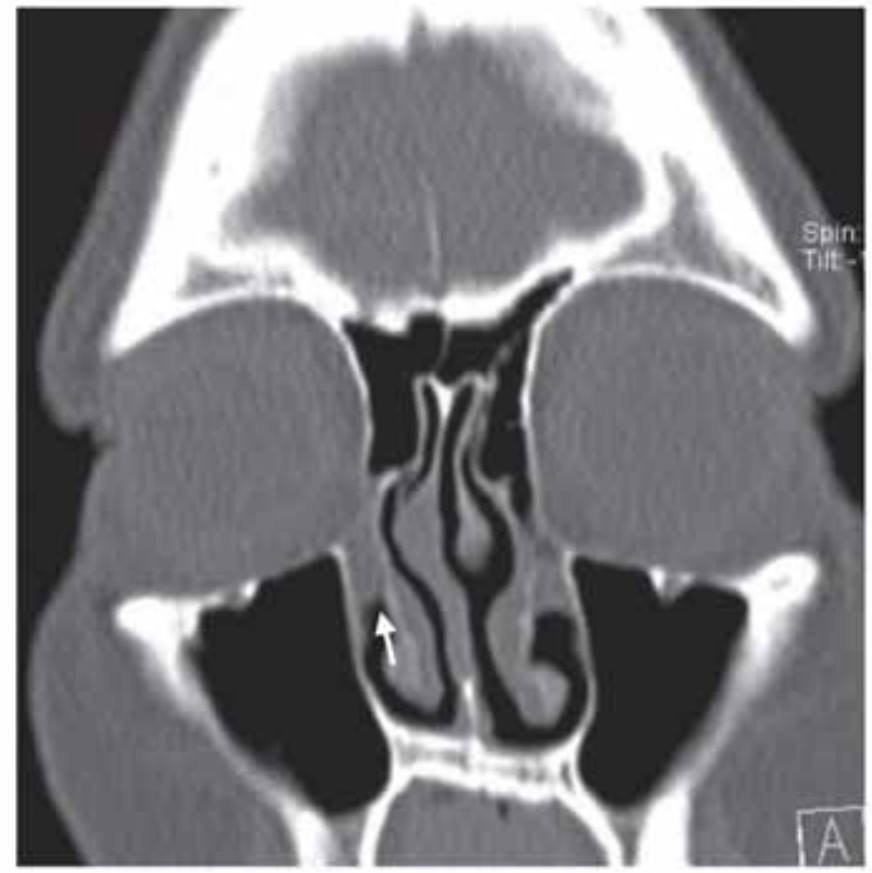

Figure 4. Coronal sinus CT scan - visualization of a lateral soft tissue fullness in the superolateral right inferior meatus (ending of the NLD) in an asymptomatic patient (white arrow). have been diagnosed by the ophthalmologic examination or a routine nasal endoscopy alone. However, the CT scan findings must be interpreted together with the ophthalmologic evaluation. Figure 4 illustrates a case where the right inferior meatus looks pathological without clinical consequences (epiphora) to the patient.

The treatment of a symptomatic dilation of the distal end of the NLD is surgical. When the cyst is small and very high in the inferior meatus, an endonasal DCR is recommended. Our case 1 received this type of treatment. Indeed, in such a case, inferior meatal surgery would have been performed in a very narrow area with instrumentation not completely adapted for such a procedure and with an increased risk of synechiae, postoperative scarring or closure of the stoma. Conversely, when the lesion is significant as for a dacryocystocele in newborns, endonasal and endoscopic marsupialisation of the nasal expansion using a microdebrider or small through-cutting instruments is the treatment of choice. This procedure has a very low morbidity, is easy and fast to perform and does not warrant nasal packing. That was how our remaining 2 patients were managed. Intraoperative syringing of the lacrimal system is mandatory to rule out any concomitant obstruction of the high lacrimal excretory system. Intubation is controversial and left to the surgeon's discretion and judgment. We opted to use a Monocath silicone catheter for a period of 2 weeks. Such practice does not seem absolutely necessary in case of a pure low obstruction but it may nevertheless increase the chance of operative success.

Postoperatively, it is common to prescribe steroids eye drops for a period of 2 weeks in combination with nasal douches.

\section{Conclusion}

Cystic dilation of the distal end of the NLD is an underestimated cause of epiphora in adults because it remains undetected by the sole ophthalmologic work-up and routine nasal endoscopy. Yet such a disease is easily diagnosed with a coronal sinus CT scan and with a nasal endoscopy of the inferior meatus. These 2 paraclinical investigations must be performed when a low obstruction of the lacrimal excretory system is suspected and their findings integrated with the ophthalmologic work-up and the patient's presenting complaints.

The treatment of such an abnormality of the lacrimal system is surgical. When the cyst is very small, an endonasal DCR is the option. However, when the cyst is significant, endoscopic marsupialisation of the nasal expansion is the preferred choice. This surgery can be performed on an ambulatory setting with low morbidity and quick rehabilitation.

ENT can play a major role in the preoperative assessment and treatment of low obstructions of the lacrimal excretory system.

\section{Financial disclosure information}

No financial disclosure.

\section{Conflict of interest}

None. 


\section{References}

1. Yung MW, Hardman-Lea S. Analysis of the results of surgical endoscopic dacryocystorhinostomy: effect of the level of obstruction. Br J Ophthalmol. 2002; 86: 792794.

2. DelGaudio JM, Wojno T. Nasolacrimal duct orifice cysts in adults: a previously unrecognized, easily treatable cause of epiphora. Laryngoscope. 2007; 117: 1830-1833.

3. Rogers GA, Murchison AP, Wojno TH, DelGaudio JM. Inferior meatus endoscopy and directed treatment for epiphora: early experience with a novel approach. Otolaryngol Head Neck Surg. 2009; 140:579584.

4. Shekunov J, Griepentrog GJ, Diehl NN, Mohney BG. Prevalence and clinical characteristics of congenital dacryocystocele. J Aapos. 2010; 14: 417-420.

5. Yee SW, Seibert RW, Bower CM, Glasier CM. Congenital nasolacrimal duct mucocele: a cause of respiratory distress. Int J Pediatr Otorhinolaryngol. 1994; 29: 151-158.

6. Mazzara CA, Respler DS, Jahn AF. Neonatal respiratory distress: sequela of bilateral nasolacrimal duct obstruction. nt J Pediatr Otorhinolaryngol. 1993; 25: 209-216.

7. Leonard DS, O'Keefe M, Rowley H, Hughes JP. Neonatal respiratory distress secondary to bilateral intranasal dacryocystocoeles. Int J Pediatr Otorhinolaryngol. 2008; 72: 1873-1877.

8. Becker BB. The treatment of congenital dacryocystocele. Am J Ophthalmol. 2006;
142: 835-838.

9. Roy D, Guevara N, Santini J, Castillo L. Endoscopic marsupialization of congenital nasolacrimal duct cyst with dacryocoele. Clin Otolaryngol Allied Sci. 2002; 27: 167170.

10. Hupin C, Leveque N, Eloy P, Bertrand B, Rombaux P. Congenital dacryocystocele: five clinical cases. B-ENT. 2008; 4: 141-145.

11. Dogan E, Yuksel NG, Ecevit MC, et al. Microdebrider assisted endoscopic marsupialization of congenital intranasal nasolacrimal duct cysts. Int J Pediatr Otorhinolaryngol. 2012; 76: 488-491.

12. Bartley GB. Acquired lacrimal drainage obstruction: an etiologic classification system, case reports, and a review of the literature. Part 1. Ophthal Plast Reconstr Surg. 1992; 8: 237-242.

13. Francisco FC, Carvalho AC, Francisco VF, Francisco MC, Neto GT. Evaluation of 1000 lacrimal ducts by dacryocystography. $\mathrm{Br} J$ Ophthalmol. 2007; 91: 43-46.

14. Zapala J, Bartkowski AM, Bartkowski SB. Lacrimal drainage system obstruction: management and results obtained in 70 patients. J Craniomaxillofac Surg. 1992; 20: 178-183.

15. Wormald PJ. Powered endoscopic dacryocystorhinostomy. Laryngoscope. 2002; 112: 69-72.

16. 16. Bloching MB, Prescher J. Treatment of lacrimal stenosis. Causes, diagnostics, and surgical procedures. Ophthalmologe. 2009; 106: 217-222
17. Sasaki T, Nagata Y, Sugiyama K. Nasolacrimal duct obstruction classified by dacryoendoscopy and treated with inferior meatal dacryorhinotomy. Part I: Positional diagnosis of primary nasolacrimal duct obstruction with dacryoendoscope. Am J of Ophthalmol. 2005; 140: 1065-1069.

18. Orhan M, Ikiz ZA, Saylam CY. Anatomical features of the opening of the nasolacrimal duct and the lacrimal fold (Hasner's valve) for intranasal surgery: a cadaveric study. Clin Anat. 2009; 22:925-931.

19. Soyka MB, Treumann T, Schlegel CT. The Agger Nasi cell and uncinate process, the keys to proper access to the nasolacrimal drainage system. Rhinology. 2010; 48: 364 367.

\section{Philippe Eloy}

HNS \& ENT Department

CHU-Mont-Godinne, UCL

Avenue Thérasse 1

5530 Yvoir

Belgium

E-mail: philippe.eloy@uclouvain.be

Tel: +32-8-142 3705

Fax: +32-8-142 3703 